\title{
Prior EGFR tyrosine-kinase inhibitor therapy did not influence the efficacy of subsequent pemetrexed plus platinum in advanced chemonaïve patients with EGFR-mutant lung adenocarcinoma
}

\author{
This article was published in the following Dove Press journal: \\ OncoTargets and Therapy \\ 23 May 2014 \\ Number of times this article has been viewed
}

\author{
Jeng-Sen Tseng',2 \\ Tsung-Ying Yang ${ }^{2}$ \\ Kun-Chieh Chen ${ }^{1,2}$ \\ Kuo-Hsuan Hsu ${ }^{1,3}$ \\ Chong-Jen $\mathrm{Yu}^{4}$ \\ Wei-Yu Liao ${ }^{4}$ \\ Chi-Ren Tsai ${ }^{5,6}$ \\ Meen-Hsin Tsai ${ }^{2,7}$ \\ Sung-Liang $\mathrm{Yu}^{8-11}$ \\ Kang-Yi Su ${ }^{8,12}$ \\ Jeremy JW Chen \\ Hsuan-Yu Chen ${ }^{7}$ \\ Gee-Chen Chang',2,13-15
}

'Institute of Biomedical Sciences, National ChungHsing University, ${ }^{2}$ Division of Chest Medicine, Department of Internal Medicine, Taichung

Veterans General Hospital, ${ }^{3}$ Division of Critical Care and Respiratory Therapy, Department of Internal Medicine, Taichung Veterans General

Hospital, Taichung, ${ }^{4}$ Department of Internal

Medicine, National Taiwan University Hospital and

National Taiwan University College of Medicine,

Taipei, ${ }^{5}$ Department of Pediatrics, Taichung

Veterans General Hospital, 'Institute of Molecular

Biology, National Chung-Hsing University,

Taichung, ${ }^{7}$ Institute of Statistical Science, Academia

Sinica, ${ }^{8}$ Department of Clinical Laboratory

Sciences and Medical Biotechnology, College of

Medicine, National Taiwan University, ${ }^{9}$ Center for

Optoelectronic Biomedicine, College of Medicine,

National Taiwan University, ${ }^{10} \mathrm{Graduate}$ Institute of

Pathology, College of Medicine, National Taiwan

University, "Department of Laboratory Medicine,

National Taiwan University Hospital, ${ }^{12}$ Center of

Genomic Medicine, National Taiwan University,

Taipei, ${ }^{13}$ School of Medicine, China Medical

University, ${ }^{14}$ Comprehensive Cancer Center,

Taichung Veterans General Hospital, Taichung,

${ }^{15}$ Faculty of Medicine, School of Medicine, National

Yang-Ming University, Taipei, Taiwan

Correspondence: Gee-Chen Chan

Faculty of Medicine, School of Medicine,

National Yang-Ming University,

155 Section 2 Linong Street, Taipei I 12, Taiwan

Tel +886 423592525 ext 3250

Fax +886 423552590

Email august@vghtc.gov.tw
Background: Tumor cells before and after epidermal growth-factor receptor (EGFR) tyrosine-kinase inhibitor (TKI) therapy might display different characteristics. The aim of this study was to evaluate the influence of prior EGFR TKI therapy on the efficacy of subsequent pemetrexed plus platinum (PP) in advanced chemonaïve patients with $E G F R$-mutant lung adenocarcinoma.

Materials and methods: Advanced chemonaïve patients with EGFR-mutant lung adenocarcinoma receiving PP as first-line chemotherapy were enrolled retrospectively in two medical centers of Taiwan. The objective of this study was to compare objective response rate (ORR), disease-control rates (DCR), progression-free survival (PFS), and overall survival (OS) of PP in patients with and without prior EGFR TKI therapy.

Results: In total, 105 patients were analyzed. Sixty-one patients (58.1\%) had prior EGFR TKI therapy and used PP as second-line treatment. The other 44 patients $(41.9 \%)$ received PP as first-line therapy. ORRs of PP in patients with and without prior EGFR TKI therapy were $24.6 \%$ and $38.6 \%$, respectively $(P=0.138)$. DCRs of the two groups were $62.3 \%$ and $65.9 \%$, respectively $(P=0.837)$. The median PFS (6.1 versus 6.1 months, $P=0.639)$ and OS (34.4 versus 32.3 months, $P=0.394)$ were comparable between the groups with and without prior EGFR TKI therapy. In a subgroup analysis of patients with prior EGFR TKI therapy, there was no significant association between the efficacy of first-line EGFR TKI and the outcome of subsequent PP therapy.

Conclusion: Our results suggested that prior EGFR TKI therapy would not influence the efficacy of subsequent PP therapy in advanced chemonaïve patients with EGFR-mutant lung adenocarcinoma.

Keywords: non-small-cell lung cancer, epidermal growth-factor receptor mutation, epidermal growth-factor receptor tyrosine-kinase inhibitor, pemetrexed

\section{Introduction}

In the last decade, many studies focused on defining the individualization of non-smallcell lung cancer (NSCLC) therapy. Both histologic and molecular subtyping have been recognized as important predictive factors in NSCLC treatment. ${ }^{1,2}$ Several Phase III studies disclosed the significantly higher response rate and longer progression-free survival (PFS) of epidermal growth-factor receptor (EGFR) tyrosine-kinase inhibitor (TKI) therapy compared with chemotherapy as first-line treatment in patients with EGFR-mutant NSCLC. ${ }^{3-6}$ 
However, studies on EGFR TKI in the first-line setting did not show significant overall survival (OS) benefit. ${ }^{7}$

Pemetrexed plus platinum (PP) is one of the standard frontline therapies for chemonaïve patients with advanced lung adenocarcinoma. In 2008, Scagliotti et al compared firstline pemetrexed plus cisplatin to gemcitabine plus cisplatin in chemonaïve patients with advanced NSCLC, and demonstrated a survival benefit of pemetrexed/cisplatin in patients with nonsquamous histology. ${ }^{8}$ The efficacy of PP for patients with EGFR-mutant lung adenocarcinoma was even better than nonselected patients, as shown in a recent Phase III study. ${ }^{9}$ However, all the reported efficacy of PP was confined to the treatment-naïve cohort, and whether the results can be applied to patients with prior EGFR TKI therapy is still unclear.

Many recent studies suggest EGFR TKI as first-line therapy for patients with EGFR-mutant lung adenocarcinoma, and PP may be reserved for patients experiencing progression of first line EGFR TKI.$^{10}$ However, the mechanism of EGFR TKI resistance is complex, ${ }^{11}$ and the post-EGFR TKI-treatment cancer cells may display different characteristics compared with the treatment-naïve cells. It is unclear if clinical resistance to EGFR TKI might also confer resistance to subsequent PP therapy. We conducted this study to evaluate whether prior EGFR TKI use influences the efficacy of subsequent PP in advanced chemonaïve patients with EGFR-mutant lung adenocarcinoma.

\section{Materials and methods Patients}

This was a retrospective study consisting of patients with advanced lung adenocarcinoma harboring EGFR mutations and treated with PP as first-line chemotherapy regimen at two medical centers of Taiwan (Taichung Veterans General Hospital and National Taiwan University Hospital) from May 2007 to June 2013. We included lung cancer patients with histologically or cytologically confirmed and inoperable lung adenocarcinoma, known EGFR mutations, and clinically measurable disease. Patients were excluded if they had only evaluable lesions, other active malignancy, prior history of other chemotherapies, incomplete data records, or received other treatments, such as radiotherapy, concurrently. Tumor, node and metastases (TNM) staging was done according to the seventh edition of AJCC Cancer Staging Handbook. ${ }^{12}$ This study was approved by the institutional review board of each institute.

\section{Data records and response evaluation}

Clinical data for analysis included patients' age, sex, Eastern Cooperative Oncology Group performance status (ECOG PS), tumor stage, smoking status, EGFR-mutation status, prior EGFR TKI treatment, and PP-treatment history. Chest computed tomographies, which were followed up with an interval of 8-12 weeks and included the liver and adrenal glands, and other required image studies for response evaluation were reviewed by two chest physicians. Unidimensional measurements as defined by Response Evaluation Criteria in Solid Tumors (RECIST) version 1.1 were used in this study. ${ }^{13}$ The objective of the study was to compare the objective response rate (ORR), disease-control rate (DCR), PFS, and OS of PP treatment in patients with and without prior EGFR TKI therapy. OS was determined from the date of the start of treatment (EGFR TKI in the group with prior EGFR TKI therapy, and PP in the group without prior EGFR TKI therapy) until the date of death, irrespective of cause.

\section{EGFR-mutation tests}

EGFR-mutation analyses using either direct sequencing or protein nucleic acid-locked nucleic acid polymerase chain reaction (PNA-LNA PCR) clamp methods were performed in patients with adequate specimens. Different methods were used in this study, because we set up a direct-sequencing method for EGFR-mutation analysis starting in 2007 and then shifted to the PNA-LNA PCR clamp in late 2009. Informed consent was obtained from all subjects.

Tumor specimens were procured for EGFR-mutation analysis as previously described. ${ }^{14}$ Briefly, deoxyribonucleic acid (DNA) was extracted from the tumors using a QIAmp DNA minikit (Qiagen, Venlo, the Netherlands) following the manufacturer's protocols. For direct sequencing, the tyrosine-kinase domain of the EGFR-coding sequence-exons 18, 19, 20, and 21 - was amplified by PCR and sequenced bidirectionally with an ABI Prism ${ }^{\circledR} 3730$ DNA analyzer (Life Technologies, Carlsbad, CA, USA) following standard protocol. For the PNA-LNA PCR clamp, real-time amplification monitoring was done using a SmartCycler ${ }^{\circledR}$ (Cepheid, Sunnyvale, CA, USA) to detect mutations on the tyrosine-kinase domain of the EGFR-coding sequence: exons $18,19,20$, and 21 .

\section{Statistical methods}

Patients were divided into without-prior EGFR TKI and with-prior EGFR TKI groups. Student's $t$-test was used to compare ages between the two groups. Other univariate analysis comparing clinical characteristics and history of prior EGFR TKI therapy on ORR and DCR was performed by exact test. Multivariate analyses using the logistic regression model with stepwise selection method were performed for ORR and DCR. The Kaplan-Meier method was used to 
estimate PFS and OS. Differences in survival time regarding prior EGFR TKI therapy were analyzed using the log-rank test. Multivariate analyses using the Cox proportional hazard model with stepwise selection method were performed for PFS and OS. All statistical tests were done with SAS version 9.1 software (SAS Institute, Cary, NC, USA). Two-tailed tests and $P$-values $<0.05$ for significance were used.

\section{Results}

\section{Patient characteristics}

There was a total of 351 patients with advanced lung adenocarcinoma and history of PP treatment from May 2007 to June 2013. A total of 163 patients were excluded, including 85 with prior history of other chemotherapies, 62 without adequate tissue for $E G F R$-mutation analysis, 12 with incomplete chart records or without measurable lesions, and four who were receiving concurrent treatments (two radiotherapy, one bevacizumab, and one erlotinib). Of the remaining 188 patients, 105 patients had detectable EGFR mutations, and 83 did not. These 105 cases were included for analysis of PP efficacy.

The baseline characteristics are shown in Table 1. Patients without prior EGFR TKI therapy were older than those with prior EGFR TKI therapy (61.6 versus 57.2 years), but this was not statistically significant $(P=0.054)$. Otherwise, there were no significant differences in baseline characteristics between the two groups.

\section{Efficacy of pemetrexed plus platinum chemotherapy}

The results of best tumor response and PFS are shown in Table 2. Of the overall 105 patients, 32 patients achieved a partial response, and 35 patients had stable disease. No patients achieved a complete response. The ORR and DCR of the overall population were $30.5 \%$ and $63.8 \%$, respectively. Survival data were followed up until the end of November 2013. At the end of data cutoff, five patients (two partial response and three stable disease, total 7.1\%) had not experienced progression, and 61 patients were still alive at the last observation, and their data were therefore censored. The median PFS was 6.1 (95\% confidence interval [CI] 5.2-7.0) months. The median OS was $32.3(95 \%$ CI 26.7-37.8) months.

ORRs of patients with and without prior EGFR TKI therapy were $24.6 \%$ and $38.6 \%$, respectively $(P=0.138)$. DCRs in the two groups were $62.3 \%$ and $65.9 \%$,

Table I Baseline demographic and clinical characteristics

\begin{tabular}{|c|c|c|c|c|}
\hline Characteristics & $\begin{array}{l}\text { Overall } \\
(n=105)\end{array}$ & $\begin{array}{l}\text { Without prior } \\
\text { EGFR TKI }(n=44)\end{array}$ & $\begin{array}{l}\text { With prior } \\
\text { EGFR TKI }(n=6 I)\end{array}$ & $P$-value ${ }^{b}$ \\
\hline Age, years, mean $\pm S D$ & $59.0 \pm 11.6$ & $61.6 \pm 11.9$ & $57.2 \pm 11.2$ & 0.054 \\
\hline Sex, n (\%) & & & & 0.552 \\
\hline Male & $46(43.8)$ & $21(47.7)$ & $25(4 I .0)$ & \\
\hline Female & $59(56.2)$ & $23(52.3)$ & $36(59.0)$ & \\
\hline ECOG PS, n (\%) & & & & 0.218 \\
\hline 0 & $36(34.3)$ & II (25.0) & $25(4 I .0)$ & \\
\hline 1 & $54(5 \mathrm{I} .4)$ & $27(61.4)$ & $27(44.3)$ & \\
\hline 2 & $14(13.3)$ & $6(13.6)$ & $8(13.1)$ & \\
\hline 3 & I (I.0) & 0 & $\mathrm{I}(\mathrm{I} .6)$ & \\
\hline Stage, n (\%) & & & & 0.173 \\
\hline IIIB & $2(1.9)$ & $2(4.5)$ & 0 & \\
\hline IV & $103(98.1)$ & $42(95.5)$ & $61(100)$ & \\
\hline Smoking status, n (\%) & & & & 0.496 \\
\hline NS & $80(76.2)$ & $32(72.7)$ & $48(78.7)$ & \\
\hline $\mathrm{C} / \mathrm{FS}$ & $25(23.8)$ & $12(27.3)$ & $13(2 \mid .3)$ & \\
\hline EGFR-mutation status, $\mathrm{n}(\%)^{c}$ & & & & 0.616 \\
\hline $19 \mathrm{Del}^{\mathrm{d}}$ & $55(52.4)$ & $21(47.7)$ & $34(55.7)$ & \\
\hline $\mathrm{L}^{2} 58 \mathrm{R}^{\mathrm{e}}$ & $40(38.1)$ & $19(43.2)$ & $21(47.7)$ & \\
\hline Others & $10(9.5)$ & $4(9.1)$ & $6(13.6)$ & \\
\hline Platinum & & & & I \\
\hline Cisplatin & $86(81.9)$ & $36(81.8)$ & $50(82.0)$ & \\
\hline Carboplatin & $19(18.1)$ & $8(18.2)$ & II (I8.0) & \\
\hline
\end{tabular}

Notes: ${ }^{a} 46$ with gefitinib, 13 with erlotinib, and two with afatinib; bage by Student's $t$-test, otherwise by exact test; 37 by direct sequencing and 68 by peptide nucleic acidlocked nucleic acid polymerase chain-reaction clamp; 'seven patients with complex mutations; ${ }^{e}$ five patients with complex mutations.

Abbreviations: SD, standard deviation; ECOG PS, Eastern Cooperative Oncology Group performance status; NS, nonsmoker; C/FS, current/former smoker; EGFR, epidermal growth-factor receptor; TKI, tyrosine-kinase inhibitor. 
Table 2 Best response and progression-free survival of pemetrexed plus platinum in chemonaïve, EGFR-mutant adenocarcinoma patients

\begin{tabular}{|c|c|c|c|c|}
\hline & $\begin{array}{l}\text { Overall } \\
(n=105)\end{array}$ & $\begin{array}{l}\text { Without prior } \\
\text { EGFR TKI }(n=44)\end{array}$ & $\begin{array}{l}\text { With prior } \\
\text { EGFR TKI }(n=6 I)\end{array}$ & $P$-value ${ }^{a}$ \\
\hline \multicolumn{5}{|l|}{ Best response, $\mathrm{n}(\%)$} \\
\hline Complete response (CR) & 0 & 0 & 0 & \\
\hline Partial response (PR) & $32(30.5)$ & $17(38.6)$ & $15(24.6)$ & \\
\hline Objective response rate (ORR) & $32(30.5)$ & $17(38.6)$ & $15(24.6)$ & 0.138 \\
\hline \multicolumn{5}{|l|}{$(\mathrm{ORR}=\mathrm{CR}+\mathrm{PR})$} \\
\hline Stable disease (SD) & $35(33.3)$ & $12(27.3)$ & $23(37.7)$ & \\
\hline Disease-control rate (DCR) & $67(63.8)$ & $29(65.9)$ & $38(62.3)$ & 0.837 \\
\hline \multicolumn{5}{|l|}{$(\mathrm{DCR}=\mathrm{CR}+\mathrm{PR}+\mathrm{SD})$} \\
\hline Progressive disease (PD) & $38(36.2)$ & I5 (34.I) & $23(37.7)$ & \\
\hline \multicolumn{5}{|l|}{ Survival, months $(95 \% \mathrm{Cl})$} \\
\hline Progression-free survival (PFS) ${ }^{b}$ & $6.1(5.2-7.0)$ & $6.1(4.8-7.4)$ & $6.1(4.9-7.4)$ & 0.639 \\
\hline Overall survival $(O S)^{c}$ & $32.3(26.7-37.8)$ & $32.3(21.8-42.7)$ & $34.4(28.9-39.8)$ & 0.394 \\
\hline
\end{tabular}

Notes: ${ }^{a}$ ORR and DCR by Fisher's exact test, PFS and OS by log-rank test; ${ }^{b}$ five patients (all with prior EGFR TKI) are still under pemetrexed plus platinum without PD; '6I patients were still alive at data cutoff.

Abbreviations: EGFR, epidermal growth-factor receptor; TKI, tyrosine-kinase inhibitor; $\mathrm{Cl}$, confidence interval.

respectively $(P=0.837)$. Univariate analysis for best response regarding patient characteristics and choice of platinum revealed that no factor correlated significantly with ORR or DCR (data not shown), and no covariate reached the significance level to enter the multivariate logistic regression model.

The results of survival analysis are shown in Table 2 and Figure 1. The median PFS (6.1 versus 6.1 months, $P=0.639)$ and $\mathrm{OS}$ (34.4 versus 32.3 months, $P=0.394$ ) were comparable between the groups with and without prior EGFR TKI therapy. Univariate analysis for survival regarding patient characteristics and choice of platinum revealed that no factor correlated significantly with PFS or OS (data not shown), and no covariates reached the significance level to enter the multivariate Cox proportional hazard model.

\section{Association between efficacies of prior EGFR TKI therapy and subsequent pemetrexed-plus-platinum chemotherapy}

In the EGFR-mutation analysis, 55 patients (52.4\%) had exon 19 deletion, and 40 patients (38.1\%) had L858R. The EGFR statuses of the remaining ten patients were heterogeneous, including G719A/S, L861Q, T790M, and other complex mutations. In 55 patients harboring exon 19 deletion, seven had complex mutations, including four with T790M. In 40 patients harboring L858R, five revealed complex mutations, including two with T790M. In 61 patients with EGFR TKI as
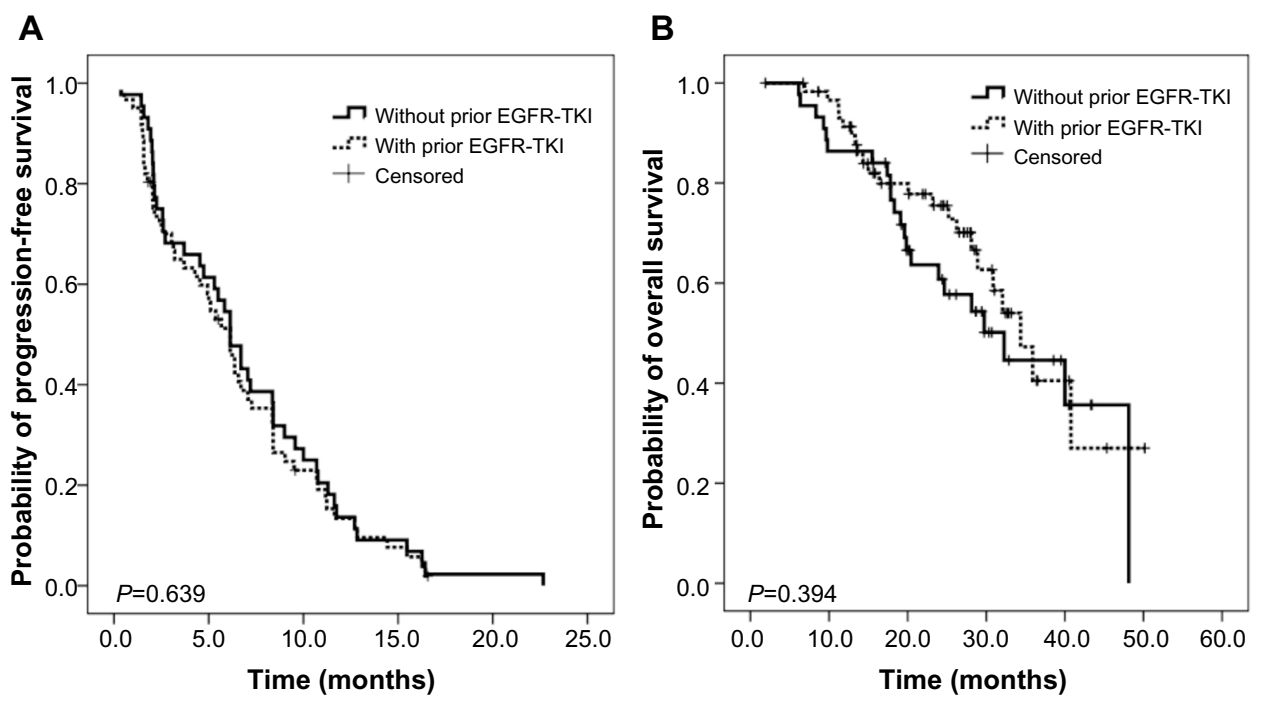

Figure I Kaplan-Meier plot showing progression-free survival (A) and overall survival (B) $(n=105)$.

Abbreviations: EGFR, epidermal growth-factor receptor; TKI, tyrosine-kinase inhibitor. 
first-line therapy, 46 received gefitinib, 13 received erlotinib, and two received afatinib. The ORR and DCR of EGFR TKI were $67.2 \%$ (47 of 61 ) and $90.2 \%$ (55 of 61 ), respectively. The median PFS of EGFR TKI was 8.0 (95\% CI 5.6-10.4) months. EGFR TKI therapy was stopped in 58 patients (95.1\%) due to disease progression. Another three patients withdrew from EGFR TKI therapy because of hepatotoxicity $(n=2)$ and financial considerations ( $n=1)$, respectively.

Results of univariate analysis for ORR and DCR in subgroups of patients with prior EGFR TKI therapy are shown in Table 3. Neither responses nor PFS of prior EGFR TKI therapy significantly influenced the efficacy of subsequent PP therapy.

\section{Subsequent treatments after pemetrexed-plus-platinum chemotherapy}

In the group treated with PP initially ( $n=44)$, two patients did not receive further treatment, one patient received other chemotherapies and 41 patients received EGFR TKI as secondline therapy. Eight of the 41 patients were still undergoing

Table 3 Univariate analysis of response rate and disease-control rate in subgroup patients with prior EGFR TKI therapy

\begin{tabular}{|c|c|c|c|c|c|}
\hline Characteristics & $\mathbf{n}$ & ORR (\%) & $P$-value & DCR (\%) & $P$-value ${ }^{a}$ \\
\hline Sex & & & 0.557 & & 0.283 \\
\hline Male & 25 & 20.0 & & 72.0 & \\
\hline Female & 36 & 27.8 & & 55.6 & \\
\hline Age (years) & & & 0.718 & & 0.336 \\
\hline$\leq 65$ & 48 & 22.9 & & 58.3 & \\
\hline$>65$ & 13 & 30.8 & & 76.9 & \\
\hline ECOG PS & & & 0.676 & & 0.718 \\
\hline $0-1$ & 52 & 23.1 & & 63.5 & \\
\hline$\geq 2$ & 9 & 33.3 & & 55.6 & \\
\hline Smoking & & & 0.156 & & 1.000 \\
\hline NS & 48 & 29.2 & & 62.5 & \\
\hline $\mathrm{C} / \mathrm{FS}$ & 13 & 7.7 & & 61.5 & \\
\hline EGFR status & & & 1.000 & & 0.075 \\
\hline 19Del or L858R & 55 & 25.5 & & 58.2 & \\
\hline Others & 6 & 16.7 & & 100.0 & \\
\hline Platinum & & & 1.000 & & 0.511 \\
\hline Cisplatin & 50 & 24.0 & & 60.0 & \\
\hline Carboplatin & II & 27.3 & & 72.7 & \\
\hline \multicolumn{6}{|c|}{ EGFR TKI therapy } \\
\hline Best response ${ }^{b}$ & & & 1.000 & & 0.417 \\
\hline Responders & 41 & 24.4 & & 58.5 & \\
\hline Nonresponders & 20 & 25.0 & & 70.0 & \\
\hline EGFR TKI PFS & & & 1.000 & & 0.567 \\
\hline$\leq 9$ months & 43 & 25.6 & & 65.1 & \\
\hline$>9$ months & 18 & 22.2 & & 55.6 & \\
\hline
\end{tabular}

Notes: ${ }^{a}$ By Fisher's exact test; 'besponders - patients with partial response, nonresponders - patients with stable or progressive disease.

Abbreviations: ORR, objective response rate; DCR, disease-control rate; ECOG PS, Eastern Cooperative Oncology Group performance status; NS, nonsmoker; C/FS, current/former smoker; EGFR, epidermal growth-factor receptor; TKI, tyrosine-kinase inhibitor; PFS, progression-free survival.
EGFR TKI therapy without PD, and 27 of the remaining 33 patients $(81.8 \%)$ received at least one subsequent therapy after progression to second-line EGFR TKI. In the group with prior EGFR TKI therapy ( $n=61)$, five patients were still undergoing PP therapy without PD, and 53 of the remaining 56 patients $(94.6 \%)$ received at least one subsequent therapy after progression to PP.

\section{Discussion}

Chemotherapy with a PP regimen is one of the standard frontline therapies for patients with advanced nonsquamous NSCLC. Mutation of the EGFR gene, a member of the ErbBreceptor family, is the most common genetic alteration in lung adenocarcinoma of East Asians. ${ }^{15}$ Therefore, several studies have tried to evaluate the association between EGFR status and the efficacy of pemetrexed. ${ }^{16-18}$ However, the baseline characteristics of most studies, including histology and concurrent platinum and treatment lines, were not homogeneous and the results were inconsistent. Furthermore, few studies have looked at the effectiveness of chemotherapy after EGFR TKI in EGFR-mutant NSCLC. ${ }^{19,20}$ In the present study, our patients were relatively homogeneous, because all were chemonaïve, had EGFR-mutant adenocarcinoma, and received platinum-containing doublet chemotherapy. Our results suggested that prior EGFR TKI therapy would not influence the efficacy of subsequent PP therapy in chemonaïve patients with advanced EGFR-mutant lung adenocarcinoma.

In the present study, we showed an ORR of $30.5 \%$, a DCR of $63.8 \%$, a median PFS of 6.1 months, and a median OS of 32.3 months with PP therapy in the 105 advanced chemonaïve patients with EGFR-mutant adenocarcinoma. The efficacy was similar to that of a Phase III study conducted by Scagliotti et al, who reported the ORR was $30.6 \%$ and the PFS was 4.8 months for all NSCLC ${ }^{8}$ In comparison, our cohort comprised only EGFR-mutant adenocarcinoma, and half of them received EGFR TKI prior to PP. The efficacy of PP was similar in both groups, and patients who received PP as first-line therapy $(n=44)$ had an ORR of $38.6 \%$ and a median PFS of 6.1 months. The results were comparable with those reported in LUX-Lung 3 study, which compared the efficacy of afatinib and pemetrexed plus cisplatin as first-line treatment for patients with advanced lung adenocarcinoma harboring EGFR mutations. ${ }^{9}$ In the chemotherapy arm, the ORR was $23 \%$ and the median PFS was 6.9 months.

Whether EGFR TKI should precede or be followed by chemotherapy is still unclear. Several studies have suggested that chemotherapy and EGFR TKI may influence the efficacy 
of each other, and drugs used in frontline therapy, whether EGFR TKI or chemotherapy, may destroy more tumor cells. In 2006, Chang et al showed that chemonaïve patients had a higher response rate to gefitinib than chemotherapy-treated patients, and hypothesized that tumor cells will evolve into a more heterogeneous and resistant phenotype with a longer time after diagnosis. ${ }^{21}$ Recently, Bai et al also showed that chemotherapy may reduce $E G F R$-mutation frequency in both plasma and tumor tissue, and suspected a reduction of overall clinical benefit of subsequent EGFR TKI after chemotherapy. ${ }^{22}$ However, in the present study, we showed that neither responses nor survival time of PP therapy were significantly different between patients with and without prior EGFR TKI therapy. As PFS represents the duration of tumor control of an investigational therapy, PFS together with ORR and DCR are important indicators to demonstrate the effectiveness of the study regimen irrespective of subsequent treatment, ${ }^{23}$ and all these outcome parameters were similar in each group of the present study. By contrast, OS represents the greatest clinical benefit but could be skewed by the effects of subsequent therapies. In the group with PP as first-line therapy, 41 of 44 (93.2\%) received EGFR TKI in subsequent therapies, which might explain the similar OS of both groups. The ORR of second-line EGFR TKI among 35 patients with measurable disease was $62.9 \%$, which was similar to that of patients receiving EGFR TKI as first-line therapy $(P=0.663)$.

In 2008, Deng et al used the lung adenocarcinoma cell lines PC9 and PC9/G with acquired resistance to gefitinib to explore the influence of acquired resistance of EGFR TKI on the sensitivity of tumor cells to chemotherapeutic drugs, and showed that no significant difference of sensitivity to pemetrexed was found between these two cell lines. ${ }^{24}$ Furthermore, a study by Maemondo et al that compared the efficacy of gefitinib and carboplatin plus paclitaxel as firstline treatment for patients with advanced NSCLC harboring EGFR mutations also showed a similar response rate of carboplatin plus paclitaxel either in the first-line setting or as subsequent therapy after progression to first-line gefitinib (30.7\% versus $28.8 \%$, respectively). ${ }^{3}$ In the gefitinib group, $67.5 \%$ of patients received carboplatin plus paclitaxel as second-line therapy. We suggest that there might be no clinically meaningful interference between EGFR TKI and chemotherapy.

In a study by Sun et al, there was no significant association between the efficacy of prior EGFR TKI and subsequent pemetrexed therapy. ${ }^{25}$ However, the results might have been limited by the diversity of previous treatments and the lack of EGFR-mutation analysis. The present study may provide a more solid result, because all patients with prior EGFR TKI therapy received PP therapy as second-line therapy, and all of them harbored EGFR mutations. Our results suggested that neither efficacy of prior EGFR TKI therapy nor the EGFR-mutation type would influence the outcome of subsequent PP therapy.

There are three limitations of this study. First, this was a retrospective study. Although data were collected retrospectively, we tried to ensure the validity of patients' characteristics, and excluded patients with confounding factors that could lead to incorrect response evaluation. A prospective trial is needed to evaluate the extent of the impact of interaction between EGFR TKI and PP in advanced EGFR-mutant lung adenocarcinoma patients. Second, the EGFR status in this study was not assessed by the same method. However, both direct sequencing and PNA-LNA PCR clamp are standard methods for EGFR testing, and this did not likely influence our results. The relatively shorter PFS of EGFR TKI in this study might be explained by the effect of complex and uncommon mutations. ${ }^{26}$ Third, there was no biological analysis in the present study, such as thymidylate synthase-expression level between the treatment-naïve and post-EGFR TKI tumor tissue, which has been recognized to be associated with pemetrexed efficacy. ${ }^{27}$

\section{Conclusion}

In conclusion, our results suggested that the efficacy of PP therapy in chemonaïve patients with $E G F R$-mutant adenocarcinoma was comparable between patients with and without prior EGFR TKI therapy. Furthermore, in patients with prior EGFR TKI therapy, there was no significant association between the efficacy of first-line EGFR TKI and the outcome of subsequent PP therapy.

\section{Acknowledgments}

We would like to thank the Comprehensive Cancer Center and Clinical Informatics Research and Development Center of Taichung Veterans General Hospital for the assistance of data collection and management, and acknowledge the technical services provided by the Pharmacogenomics Lab of the National Research Program for Biopharmaceuticals (NRPB) (TR6-3) and Integrated Core Facility for Functional Genomics of the National Core Facility Program for Biotechnology (NCFPB) (C5), which were supported by the National Science Council (NSC102-2325-B-002-078 and NSC102-2319-B-002).

\section{Disclosure}

The authors have no conflicts of interest in this work. 


\section{References}

1. West H, Harpole D, Travis W. Histologic considerations for individualized systemic therapy approaches for the management of non-small cell lung cancer. Chest. 2009;136(4):1112-1118.

2. Moreira AL, Thornton RH. Personalized medicine for non-small-cell lung cancer: implications of recent advances in tissue acquisition for molecular and histologic testing. Clin Lung Cancer. 2012;13(5): 334-339.

3. Maemondo M, Inoue A, Kobayashi K, et al. Gefitinib or chemotherapy for non-small-cell lung cancer with mutated EGFR. $N$ Engl J Med. 2010;362(25):2380-2388.

4. Mitsudomi T, Morita S, Yatabe Y, et al. Gefitinib versus cisplatin plus docetaxel in patients with non-small-cell lung cancer harbouring mutations of the epidermal growth factor receptor (WJTOG3405): an open label, randomised phase 3 trial. Lancet Oncol. 2010;11(2):121-128.

5. Mok TS, Wu YL, Thongprasert S, et al. Gefitinib or carboplatinpaclitaxel in pulmonary adenocarcinoma. N Engl J Med. 2009;361(10): 947-957.

6. Zhou C, Wu YL, Chen G, et al. Erlotinib versus chemotherapy as first-line treatment for patients with advanced EGFR mutationpositive non-small-cell lung cancer (OPTIMAL, CTONG-0802): a multicentre, open-label, randomised, phase 3 study. Lancet Oncol. 2011;12(8):735-742.

7. Mok T, Yang JJ, Lam KC. Treating patients with EGFR-sensitizing mutations: first line or second line - is there a difference? J Clin Oncol. 2013;31(8):1081-1088.

8. Scagliotti GV, Parikh P, von Pawel J, et al. Phase III study comparing cisplatin plus gemcitabine with cisplatin plus pemetrexed in chemotherapy-naive patients with advanced-stage non-small-cell lung cancer. J Clin Oncol. 2008;26(21):3543-3551.

9. Sequist LV, Yang JC, Yamamoto N, et al. Phase III study of afatinib or cisplatin plus pemetrexed in patients with metastatic lung adenocarcinoma with EGFR mutations. J Clin Oncol. 2013;31(27):3327-3334.

10. Leighl NB. Treatment paradigms for patients with metastatic nonsmall-cell lung cancer: first-, second-, and third-line. Curr Oncol. 2012; 19 Suppl 1:S52-S58.

11. Oxnard GR, Arcila ME, Chmielecki J, Ladanyi M, Miller VA, Pao W. New strategies in overcoming acquired resistance to epidermal growth factor receptor tyrosine kinase inhibitors in lung cancer. Clin Cancer Res. 2011;17(17):5530-5537.

12. Edge SB, Byrd DR, Compton CC, Fritz AG, Greene FL, Trotti A, editors. AJCC Cancer Staging Handbook. 7th ed. New York: Springer; 2009.

13. Eisenhauer EA, Therasse P, Bogaerts J, et al. New response evaluation criteria in solid tumours: revised RECIST guideline (version 1.1). Eur J Cancer. 2009;45(2):228-247.

14. Tseng JS, Yang TY, Chen KC, Hsu KH, Chen HY, Chang GC. Retrospective study of erlotinib in patients with advanced squamous lung cancer. Lung Cancer. 2012;77(1):128-133.
15. An SJ, Chen ZH, Su J, et al. Identification of enriched driver gene alterations in subgroups of non-small cell lung cancer patients based on histology and smoking status. PloS One. 2012;7(6):e40109.

16. Wu SG, Yang CH, Yu CJ, et al. Good response to pemetrexed in patients of lung adenocarcinoma with epidermal growth factor receptor (EGFR) mutations. Lung Cancer. 2011;72(3):333-339.

17. Camidge DR, Kono SA, Lu X, et al. Anaplastic lymphoma kinase gene rearrangements in non-small cell lung cancer are associated with prolonged progression-free survival on pemetrexed. J Thorac Oncol. 2011;6(4):774-780.

18. Kim YH, Hirabayashi M, Togashi Y, et al. Phase II study of carboplatin and pemetrexed in advanced non-squamous, non-small-cell lung cancer: Kyoto Thoracic Oncology Research Group Trial 0902. Cancer Chemother Pharmacol. 2012;70(2):271-276.

19. Gridelli C, Ciardiello F, Gallo C, et al. First-line erlotinib followed by second-line cisplatin-gemcitabine chemotherapy in advanced nonsmall-cell lung cancer: the TORCH randomized trial. J Clin Oncol. 2012;30(24):3002-3011.

20. Wu JY, Shih JY, Yang CH, et al. Second-line treatments after first-line gefitinib therapy in advanced nonsmall cell lung cancer. Int J Cancer. 2010;126(1):247-255.

21. Chang GC, Tsai CM, Chen KC, et al. Predictive factors of gefitinib antitumor activity in East Asian advanced non-small cell lung cancer patients. J Thorac Oncol. 2006;1(6):520-525.

22. Bai H, Wang Z, Chen K, et al. Influence of chemotherapy on EGFR mutation status among patients with non-small-cell lung cancer. J Clin Oncol. 2012;30(25):3077-3083.

23. Soria JC, Massard C, Le Chevalier T. Should progression-free survival be the primary measure of efficacy for advanced NSCLC therapy? Ann Oncol. 2010;21(12):2324-2332.

24. Deng QF, Su B, Zhao YM, Zhou CC. [Sensitivity of two cell lines with acquired resistance to gefitinib to several chemotherapeutic drugs]. Zhonghua Zhong Liu Za Zhi. 2008;30(11):813-816. Chinese.

25. Sun JM, Oh DY, Lee SH, et al. The relationship between response to previous systemic treatment and the efficacy of subsequent pemetrexed therapy in advanced non-small cell lung cancer. Lung Cancer. 2010;68(3):427-432.

26. Wu JY, Yu CJ, Chang YC, Yang CH, Shih JY, Yang PC. Effectiveness of tyrosine kinase inhibitors on "uncommon" epidermal growth factor receptor mutations of unknown clinical significance in non-small cell lung cancer. Clin Cancer Res. 2011;17(11):3812-3821.

27. Christoph DC, Asuncion BR, Hassan B, et al. Significance of folate receptor alpha and thymidylate synthase protein expression in patients with non-small-cell lung cancer treated with pemetrexed. J Thorac Oncol. 2013;8(1):19-30.
OncoTargets and Therapy

\section{Publish your work in this journal}

OncoTargets and Therapy is an international, peer-reviewed, open access journal focusing on the pathological basis of all cancers, potential targets for therapy and treatment protocols employed to improve the management of cancer patients. The journal also focuses on the impact of management programs and new therapeutic agents and protocols on

\section{Dovepress}

patient perspectives such as quality of life, adherence and satisfaction. The manuscript management system is completely online and includes a very quick and fair peer-review system, which is all easy to use. Visit http://www.dovepress.com/testimonials.php to read real quotes from published authors. 